\title{
Apolipoprotein E-deficient mice created by systemic administration of antisense oligodeoxynucleotides: a new model for lipoprotein metabolism studies
}

\author{
R Morishita ${ }^{1,2}$, G H Gibbons ${ }^{3}$, Y Kaneda ${ }^{1}$, L Zhang ${ }^{3}$, T Ogihara ${ }^{2}$ \\ and $\mathbf{V} \mathbf{J}$ Dzau $^{3}$ \\ ${ }^{1}$ Division of Gene Therapy Science, Osaka University Medical School, Suita 565-0871, Japan \\ ${ }^{2}$ Department of Geriatric Medicine, Osaka University Medical School, Suita, Japan \\ ${ }^{3}$ Department of Medicine, Harvard Medical School, Brigham and Women's Hospital, Boston, Massachusetts 02115, USA \\ (Requests for offprints should be addressed to R Morishita, Division of Gene Therapy Science, Osaka University Medical School, 2-2 Yamada-oka, \\ Suita 565, Japan; Email: morishit@geriat.med.osaka-u.ac.jp)
}

\begin{abstract}
Atherosclerotic cardiovascular disease results from complex interactions among multiple genetic and environmental factors. Thus, it is important to elucidate the influence of each factor on cholesterol metabolism. For this purpose, transgenic/gene-targeting technology is a powerful tool for studying gene functions. However, this technology has several disadvantages such as being time consuming and expensive. Accordingly, we established new animal models using in vivo gene transfer technology. In this study, we examined the feasibility of the creation of a new animal model for the study of atherosclerosis. We hypothesized that apolipoprotein (apo) E-deficient mice can be created by systemic administration of antisense apo E oligodeoxynucleotides (ODN) coupled to the HVJliposome complex. Initially, we examined the localization and cellular fate of FITC-labeled antisense ODN administered intravenously. FITC-labeled ODN transfection by the HVJ-liposome method resulted in fluorescence in the liver, spleen and kidney, but not in other organs such as brain. Moreover, fluorescence with the HVJ-liposome method was sustained for up to 2 weeks after transfection, which resulted in a striking difference from transfection of ODN alone or ODN in liposomes without HVJ, which showed rapid disappearance of fluorescence (within 1 day). Given these unique characteristics of the HVJ-liposome method, we next examined transfection of antisense apo E
\end{abstract}

ODN by intravenous administration. Transfection of antisense apo E ODN resulted in a marked reduction of apo $\mathrm{E}$ mRNA levels in the liver, but no change in apo B and $\beta$-actin mRNA levels. In mice fed a normal diet, a transient increase in cholesterol and triglyceride levels was observed in the antisense apo E-treated group, but they returned to normal levels by 6 days after transfection. Similar findings were also found in mice fed a high cholesterol diet. Neither scrambled nor mismatched ODN resulted in any increase in cholesterol. To make chronic hypercholesterolemic mice, we therefore performed repeated injections of apo E antisense ODN. Whenever antisense apo E ODN were injected, mice showed a transient increase in cholesterol and triglyceride. Cumulative administration of antisense apo E ODN resulted in a sustained increase in cholesterol for up to 3 weeks after the last transfection. Finally, mice treated with repeated injections of antisense apo E every week developed sustained hypercholesterolemia and hypertriglyceridemia until withdrawal of injections. Apolipoprotein-deficient mice created by intravenous administration of antisense ODN are a promising new animal model to help understand the role of apolipoprotein in vivo and develop a new drug therapy targeting apolipoprotein.

Journal of Endocrinology (2002) 175, 475-485

\section{Introduction}

Atherosclerotic cardiovascular disease results from complex interactions among multiple genetic and environmental factors. Among these factors, apolipoprotein (apo) $\mathrm{E}$ has been of interest because of its known genetic variant (Zannis \& Breslow 1981, Utermann et al. 1982). Apo $\mathrm{E}$ is one of the apolipoproteins that coat the surface and mediate the metabolism of lipoprotein particles. It is synthesized primarily in the liver, although synthesis has been documented in the brain and other tissues. One of its most important functions is to serve as a high affinity ligand for the apo B and low density lipoprotein (LDL) receptors and for the chylomicron-remnant receptor, thereby allowing the specific uptake of apo E-containing particles by the liver. In addition to lipoprotein transport, 
apo $\mathrm{E}$ has been postulated to play a role in reverse cholesterol transport, local redistribution of cholesterol within tissues, immunoregulation, cell proliferation, and differentiation (Mahley 1988). Uniquely, a frequent genetic variant of human apo E, apo E2, differs from the most common form, apo E3 (Zannis et al. 1982). The amino acid substitution in the LDL receptor binding region reduces the binding activity of apo E2 to less than $2 \%$ relative to that of apo E3 (Weintrab et al. 1987). As a clinically important point, homozygosity for the gene apo E2 is associated with type III hyperlipoproteinemia accompanied by high cholesterol and triglyceride levels, resulting in the development of atherosclerosis (Mahley \& Rall 1989). Recent progress in molecular biology, such as transgenic technology, has enabled the study of specific gene function, which classical pharmacological experiments could not dissect out. Over-expression of the apo E gene in mice resulted in a decrease in cholesterol and prevention of atherosclerosis (Shimano et al. 1992). Recently, there has been increased interest in the role of apo $\mathrm{E}$ in cholesterol metabolism, because mice lacking the apo E gene have been created by gene targeting technology. These mice show severely high cholesterol and relatively high triglyceride levels in response to a Westerntype diet (Plump et al. 1992, Zhang et al. 1992). Although transgenic/gene targeting technology is a powerful tool for studying gene functions, this technology has several disadvantages: (1) it is time consuming and expensive, (2) it is dependent on developmental and systemic effects, and (3) it is associated with difficulty in the experimental protocol. Accordingly, we have established a new animal model using in vivo gene transfer technology (Dzau et al. 1994). For example, in vivo transfer of the renin gene directly into adult rat liver resulted in hypertension (Tomita et al. 1993), and in vivo transfer of the angiotensin converting enzyme gene into blood vessels resulted in vascular hypertropy independent of a systemic effect (Morishita et al. 1994a). In this study, we applied antisense technology to study specific gene functions, besides gene targeting technology. Here, inhibition of apolipoprotein $\mathrm{E}$ by the systemic administration of antisense oligodeoxynucleotides (ODN) using the HVJ (Hemagglutinating Virus of Japan)liposome method resulted in hypercholesterolemia and hypertriglyceridemia as a new animal model ('antigenic' model) of lipoprotein metabolism.

\section{Materials and Methods}

Synthesis of oligodeoxynucleotides and selection of target sequences

The sequences of ODN against mouse apo $\mathrm{E}$ used in this study were as follows: antisense: $5^{\prime}$-CCA-CAGAGC-CTT-CAT-CTT-C-3', sense: 5'-GAA-GATGAA-GGC-TTC-GTG-G-3' $(-4$ to +15 of the mouse sequence; these sequences are identical in mouse and human apolipoprotein E). We also synthesized scrambled and a 4-base pair mismatched ODN of the apolipoprotein E antisense ODN (scrambled ODN: 5'-CTT-CGTCGG-TAC-CGT-CTT-C-3', mismatched ODN: 5'-CCA-CAG-TGC-CCT-CGT-TTT-C-3'). Synthetic ODN were purified over a NAP 10 column (Pharmacia), and quantitated by spectrophotometry (Morishita et al. 1993, 1994b).

\section{Preparation of HVJ-liposome}

Phosphatidylserine, phosphatidylcholine, and cholesterol were mixed in a weight ratio of 1:4.8:2 (Tomita et al. 1993, Morishita et al. 1993, 1994a,b). Dried lipid was hydrated in $200 \mu \mathrm{l}$ balanced salt solution (BSS; $137 \mathrm{mM}$ $\mathrm{NaCl}, 5 \cdot 4 \mathrm{mM} \mathrm{KCl}, 10 \mathrm{mM}$ Tris-HCl, $\mathrm{pH}$ 7.6) containing ODN. The control group as a negative control did not contain ODN (BSS $200 \mu \mathrm{l}$ ). Liposomes were prepared by shaking and sonication. Purified HVJ ( $Z$ strain) was inactivated by UV irradiation $\left(110 \mathrm{erg} / \mathrm{mm}^{2} / \mathrm{s}\right)$ for $3 \mathrm{~min}$ just before use. The liposome suspension $(0.5 \mathrm{ml}$, containing $10 \mathrm{mg}$ lipid) was mixed with HVJ (10 000 hemagglutinating units) in a total volume of $4 \mathrm{ml} \mathrm{BSS}$. The mixture was incubated at $4{ }^{\circ} \mathrm{C}$ for $5 \mathrm{~min}$ and then for 30 min with gentle shaking at $37^{\circ} \mathrm{C}$. Free HVJ was removed from the HVJ-liposome by sucrose density gradient centrifugation.

\section{In vivo transfection of FITC-labeled antisense ODN}

FITC-labeled phosphorothioate antisense ODN were provided by Clontech Inc. (Palo Alto, CA, USA). FITC was used to label the $3^{\prime}$ and $5^{\prime}$ ends of the antisense apo E ODN using fluorescein-ODN phosphoramidite (Morishita et al. 1994b). Transfer of phosphorothioate ODN was performed according to three protocols: (1) $200 \mu \mathrm{l} \mathrm{HVJ}$ complex with FITC-labeled phosphorothioate ODN $(3 \mu \mathrm{M})$ was used; (2) $200 \mu \mathrm{l}$ ODN in liposomes without HVJ particles: liposome complex with ODN $(3 \mu \mathrm{M})$, but without HVJ particles was used; (3) $200 \mu \mathrm{l}$ FITC-labeled ODN $(30 \mu \mathrm{M})$ was used. Each complex was injected into mice via the tail vein. At $30 \mathrm{~min}, 12$ and $24 \mathrm{~h}$, and at 3, 7 and 14 days after transfection, mice were killed, and tissues were removed after perfusion-fixation with 4\% paraformaldehyde under physiological pressure $(110 \mathrm{mmHg})$. Sections were examined by fluorescent microscopy, after staining with erichrome black $\mathrm{T}$ solution; elastic fibers stained dark red and were readily distinguishable from the specific FITC-labeled antisense ODN (data not shown) as previously described (Kittelberger et al. 1989, Morishita et al. 1994b). We also performed quantitative measurement of fluorescence to confirm the efficiency of FITC-labeled ODN transfection with the HVJ-liposome method $(1 \mu \mathrm{M})$. FITC-labeled ODN with the HVJ-liposome complex was intravenously injected into the tail vein. For measurement of 

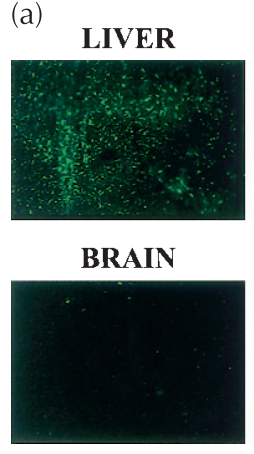

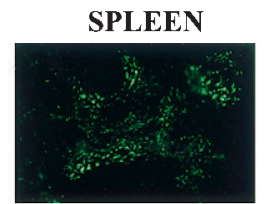

TESTIS

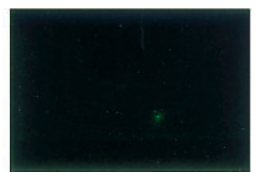

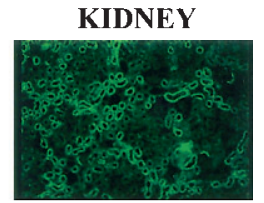

LUNG

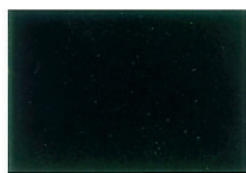

(c)

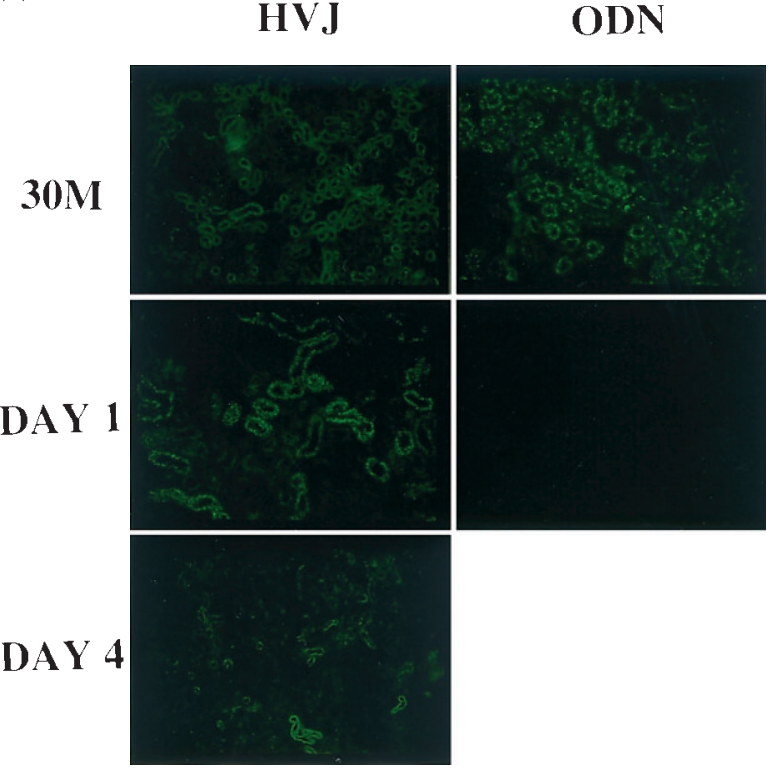

(d)

HVJ
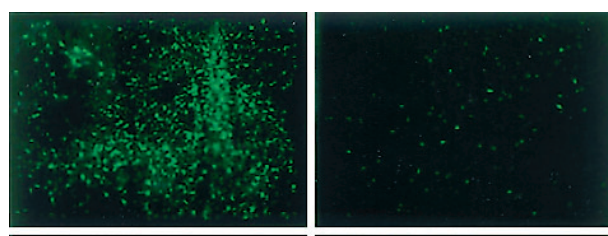

DAY 1
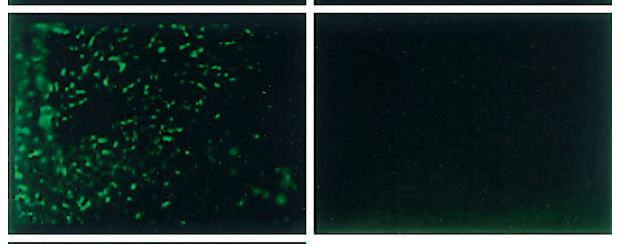

DAY 4

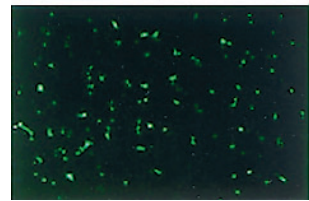

ODN

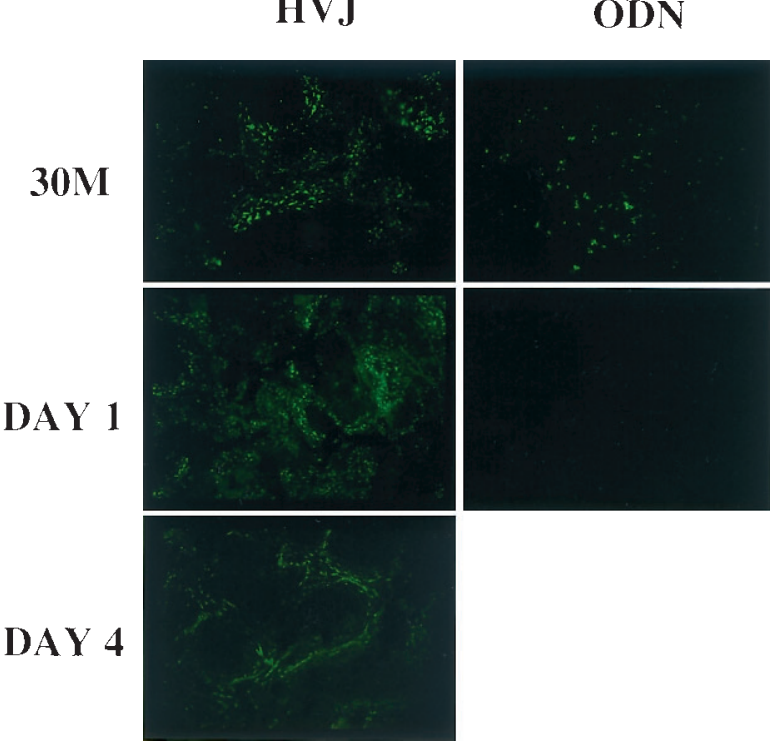

Figure 1 (a) Representative fluorescence microscopy of various organs transfected with FITC-labeled ODN by the HVJ-liposome method at 1 day after transfection. (b-d) Comparison of passive uptake and the HVJ-liposome method in liver (b), spleen (c) and kidney (d). HVJ, mice transfected with FITC-labeled ODN by the HVJ-liposome method; ODN, mice transfected with FITC-labeled ODN without the HVJ-liposome method; 30M, at 30 min after transfection; DAY 1, at 1 day after transfection; DAY 4, at 4 days after transfection.

fluorescence, tissues were then extracted at 1 day after transfection. They were homogenized in $1 \mathrm{ml}$ PBS, centrifuged and $400 \mu \mathrm{l}$ of the supernatant were used for measurement with a fluorescence meter (RF1500 spectrofluorophotometer, Shimazu) (excitation, $493 \mathrm{~nm}$; emission, $520 \mathrm{~nm}$ ).
Reverse transcription (RT)-PCR and Northern blot

First, $200 \mu \mathrm{l} \mathrm{HVJ-liposome} \mathrm{complex} \mathrm{containing} \mathrm{sense} \mathrm{or}$ antisense ODN (15 $\mu \mathrm{M}$ in liposomes/body) were injected into the tail vein of mice fed a normal or a high cholesterol diet. One day after transfection, the liver was infused with sterile PBS, then frozen immediately in liquid nitrogen 
and stored at $-80{ }^{\circ} \mathrm{C}$ until RNA extraction. RNA was extracted using RNAzol (Tel-Test Inc., Dallas, Texas, USA). Extracted RNA was quantified by measuring absorbance at $260 \mathrm{~nm}$. PCR amplification of $0.5 \mu \mathrm{g}$ aliquots of extracted RNA was performed with oligonucleotide primers complementary to the apo E gene. The $5^{\prime}$ primer was 5'-GAG-CTG-GAG-GAA-CAG-CTGGGT-3'; the $3^{\prime}$ primer was $5^{\prime}$-CTC-GAC-CTC-CTTGTC-GAC-CCA-3' (Wang et al. 1989). Primers complementary to the apo B gene were also used (the $5^{\prime}$ primer was $5^{\prime}$-GGA-ATT-CAT-TAT-CTG-AAT-GCA-TC$3^{\prime}$; the $3^{\prime}$ primer was $5^{\prime}$-GGG-ATC-CAT-GAT-TCGATC-AAT-AA-3') (Xiong et al. 1992). Extreme care was taken to avoid contamination of tissue samples with trace amounts of experimental RNA. Aliquots of RNA derived from tissues were amplified simultaneously by PCR and compared with a negative control (primers without RNA). The amplification protocol was as follows: $95{ }^{\circ} \mathrm{C}$ for $30 \mathrm{~s}, 55^{\circ} \mathrm{C}$ for $30 \mathrm{~s}, 72{ }^{\circ} \mathrm{C}$ for $1 \mathrm{~min}$. Amplification products were electrophoresed through $2 \%$ agarose gels stained with ethidium bromide. Thirty and forty cycles of PCR amplification were used (Morishita et al. 1993, 1994b). As a control for the integrity of the extracted RNA as well as for the PCR protocol, an aliquot of each RNA sample was also amplified with oligonucleotide primers complementary to the mouse $\beta$-actin gene (donated by Clontech). Extreme care was taken to avoid contamination of tissue samples with trace amounts of experimental RNA. Aliquots of RNA from both experimental and negative control tissues were amplified simultaneously, with the same reagents, by individuals who were blinded to the identity of the samples. To check if the detected band was due to the transcription of the gene, RNA samples treated with RNase A were amplified simultaneously as a negative control. These samples did not result in a visual band (data not shown). At least three aliquots of each RNA sample were subjected to separate PCR amplifications in all experiments.

We also examined apo E mRNA expression using Northern blot analysis in a standard manner (Morishita et al. 1992). The 5' PCR primer of apo E was labeled by an end-labeling kit (donated by Clontech), and used as a probe.

\section{Serum cholesterol and triglyceride levels}

Male C57/BL6 mice (12 weeks; Charles River Laboratories, Boston, MA, USA) were used for experiments. The mice were maintained and bred under conventional conditions with food and tap water available ad libitum, except on the days of blood sampling, and a $12 \mathrm{~h}$ light-12 h darkness photoperiod. Cholesterol, triglyceride and high density lipoprotein (HDL) assays were performed as recommended by the manufacturer of the assay kit (Sigma Immunochemicals, St Louis, MO, USA).

\section{Transfection of antisense ODN}

Mice transfected with sense, scrambled, mismatched or antisense ODN were examined for measurement of cholesterol and triglyceride levels. The following protocols were performed. (1) Systemic administration of ODN via the tail vein was performed on day 0 , and blood was obtained on days 1, 2, 3 and 6 after transfection. (2) ODN transfection was performed on days 0,1 , and 2 to study the cumulative effects of ODN. (3) ODN transfection was performed on days 0,7 , and 16 to study the effect of repeated injections. (4) ODN transfection was performed once or twice a week to follow the changes in serum cholesterol and triglyceride levels. After transfection, blood specimens were collected periorbitally into heparincontaining microtubes to study the time-course of changes in glucose level. Mice were fasted for at least $8 \mathrm{~h}$ before blood sampling. A normal diet (without cholesterol) or a high cholesterol diet (1\% cholesterol, $0 \cdot 2 \%$ cholic acid and $20 \%$ coconut oil; Dyets Inc., Bethlehem, PA, USA) was administered.

\section{Statistical analysis}

All values are expressed as means \pm S.E.M. Analysis of variance with Duncan's test was used to determine the significance of differences in multiple comparisons. $P<0 \cdot 05$ was considered to be statistically significant.

\section{Results}

\section{Detection of FITC-labeled antisense ODN after systemic transfection}

Initially, we studied the localization of FITC-labeled antisense ODN to evaluate the character of various ODN delivery transfer methods. At $30 \mathrm{~min}$ after transfection, fluorescence was detected in the liver, spleen and kidney (tubular cells, but not mesangial cells) with the HVJliposome method, whereas no fluorescence was observed in brain, testis, lung (Fig. 1a), heart, muscle and intestine (data not shown). In contrast, passive uptake of FITClabeled antisense ODN resulted mainly in the kidney, with little in the liver and spleen (Fig. 1b-d). In other tissues, no fluorescence was observed with passive uptake. Interestingly, a striking difference in the time course of fluorescence was observed between the HVJ-liposome method and passive uptake (Fig. 1b-d). Rapid disappearance of fluorescence (within 1 day) was found with passive uptake, whereas sustained fluorescence was observed with the HVJ-liposome method up to 2 weeks after transfection. Finally, we studied the role of HVJ particles in systemic administration of ODN. Transfection of FITC-labeled antisense ODN in the liposome complex without HVJ particles resulted in the same localization as with the HVJ-liposome method (data not shown). 
(a)
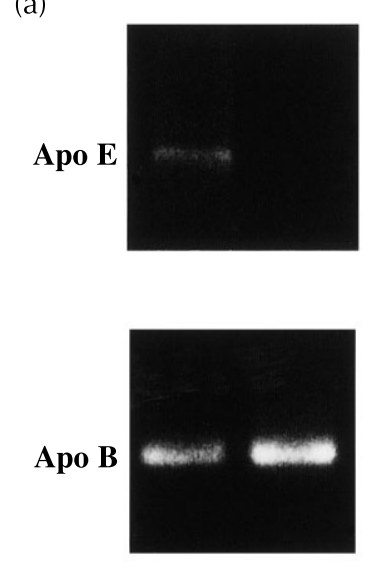

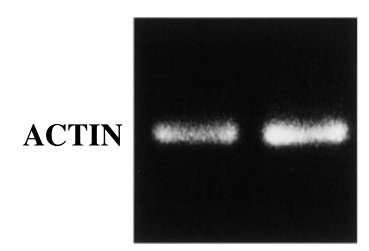

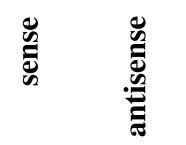

(b)

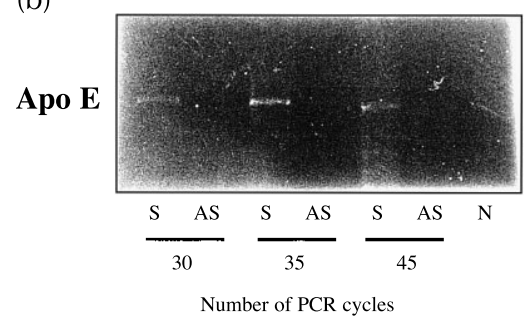

(c)

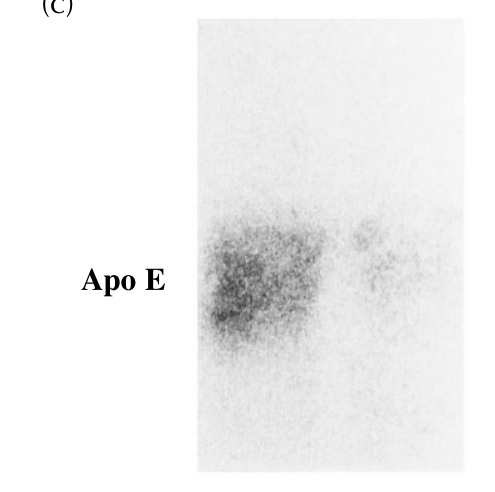

28S RNA

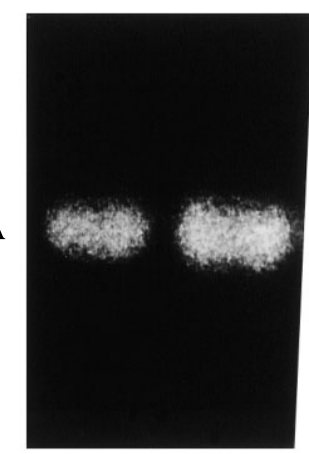

Figure 2 (a) Detection of apo E, B and $\beta$-actin gene expression in liver of sense and antisense apo E ODN-transfected mice by RT-PCR. (b) Semiquantative RT-PCR analysis of apo E mRNA in liver of sense (S) and antisense (AS) apo E ODN-transfected mice. (c) Northern blot of apo E mRNA in liver of sense and antisense apo E ODN-transfected mice. sense, RNA from the liver of mice transfected with sense apo E ODN; antisense, RNA from the liver of mice transfected with antisense apo E ODN; Apo E, apo E mRNA; Apo B, apo B mRNA; ACTIN, $\beta$-actin mRNA.

However, rapid disappearance of fluorescence was observed with the liposome-mediated method without HVJ, the same as for passive uptake. We confirmed the prolongation of ODN half-life with the HVJ-liposome method, consistent with previous reports (Morishita et al. 1994c, 1996). Untreated or HVJ complex without FITC-labeled antisense ODN resulted in no specific fluorescence (data not shown). Fluorescence resulting from FITC-labeled antisense ODN was easily distinguished from background auto-fluorescence. Systemic infusion of free FITC did not result in specific fluorescence in the tissues (data not shown), demonstrating that this fluorescence was specific for FITC-labeled ODN. Marked accumulation of ODN in the liver using the HVJ-liposome method was also confirmed by the measurement of fluorescence. Transfection of FITC-labeled ODN by the HVJ-liposome method resulted in a significant $(P<0.01$ vs other tissues) increase in fluorescence detected in the liver as compared with other tissues at 1 day after transfection (liver:
$46500 \pm 5600$, spleen: $3600 \pm 500$, kidney: $2300 \pm 400$ fluorescence intensity, heart: not detected).

\section{Specificity of antisense apo E ODN}

To study the effect of systemic administration of antisense apo E ODN, initially we performed RT-PCR of liver. RT-PCR using RNA of mice transfected with antisense apo E ODN showed no visual band at 35 cycles, whereas RT-PCR from RNA of mice transfected with sense ODN yielded a clear visual band (Fig. 2a). Semiquantitative RT-PCR also showed a consistent decrease in apo E mRNA level in antisense ODN-treated mice (Fig. 2b). Moreover, the specificity of the antisense ODN effect was supported by the observation that no significant change in apo $B$ and $\beta$-actin mRNA levels was observed in mice transfected with antisense apo E ODN (Fig. 2a). These findings were also supported by Northern blot analysis. 
Table 1 Effect of mismatched and scrambled ODN on serum cholesterol and triglyceride levels in normal mice. Values $=$ transfected mice - untransfected mice with vehicle injection and are expressed as means \pm S.E.M.

\begin{tabular}{|c|c|c|c|c|}
\hline & Antisense & Mismatched & Scrambled & Significance \\
\hline \multicolumn{5}{|c|}{ Day } \\
\hline \multicolumn{5}{|c|}{ Cholesterol } \\
\hline 1 & $20 \cdot 0 \pm 9 \cdot 7^{*}$ & $-1 \cdot 7 \pm 7 \cdot 2$ & $-3 \cdot 2 \pm 9 \cdot 1$ & ${ }^{*} P<0 \cdot 01$ \\
\hline 2 & $9 \cdot 5 \pm 4 \cdot 6^{*}$ & $0.6 \pm 2.9$ & $-2 \cdot 7 \pm 4 \cdot 7$ & ${ }^{*} P<0.01$ \\
\hline 3 & $20 \cdot 6 \pm 10 \cdot 3^{*}$ & $2 \cdot 1 \pm 9 \cdot 4$ & $-2 \cdot 1 \pm 9 \cdot 5$ & ${ }^{*} P<0.01$ \\
\hline 6 & $6 \cdot 1 \pm 3 \cdot 6$ & $3 \cdot 1 \pm 8 \cdot 1$ & $-0 \cdot 4 \pm 8 \cdot 4$ & NS \\
\hline \multicolumn{5}{|c|}{ Triglyceride } \\
\hline 1 & $18 \cdot 0 \pm 6 \cdot 2^{*}$ & $4 \cdot 3 \pm 3 \cdot 9$ & $3 \cdot 7 \pm 2 \cdot 9$ & $* P<0.01$ \\
\hline 2 & $21 \cdot 7 \pm 8 \cdot 4^{*}$ & $-2 \cdot 6 \pm 2 \cdot 0$ & $1 \cdot 0 \pm 11 \cdot 1$ & ${ }^{*} P<0.01$ \\
\hline 3 & $25 \cdot 8 \pm 7 \cdot 1^{*}$ & $7 \cdot 4 \pm 7 \cdot 3$ & $-9 \cdot 4 \pm 5 \cdot 2$ & ${ }^{*} P<0.01$ \\
\hline 6 & $4 \cdot 3 \pm 6 \cdot 6$ & $-5 \cdot 7 \pm 4 \cdot 0$ & $-10 \cdot 0 \pm 8 \cdot 2$ & NS \\
\hline
\end{tabular}

NS= not significant

Transfection of antisense apo E ODN decreased hepatic apo E mRNA levels as compared with sense ODN (Fig. 2c).

\section{Effect of systemic administration of antisense apo E ODN in} mice on a normal diet

Given that the existence of FITC-labeled antisense ODN was detected in the liver, spleen and kidney up to 2 weeks after transfection, we hypothesized that local inhibition of apo $\mathrm{E}$ in the liver by systemic transfection of antisense apo E ODN via the tail vein would modulate cholesterol metabolism. Accordingly, we studied the effect of a single systemic administration of antisense apo E ODN in mice on a normal diet. We measured serum cholesterol and triglyceride levels in mice transfected with sense and antisense apo E ODN. Cholesterol concentration was increased from day 1 to day 3 in antisense ODNtransfected mice after transfection, but on day 6 after transfection there was no significant difference between sense and antisense ODN-transfected mice (Fig. 3a,b). Similarly, serum triglyceride concentration was increased in antisense ODN-transfected mice as compared with sense ODN-transfected mice (Fig. 3c), while there was no significant change in HDL-cholesterol (data not shown). The specificity of antisense ODN was also supported by the observation that systemic administration of mismatched or scrambled ODN did not result in any significant change in cholesterol and triglyceride levels (Table 1).

\section{Effect of systemic administration of antisense apo E ODN in} mice on a high cholesterol diet

To investigate the feasibility of the antisense strategy to establish a new knockout model, we studied the effect of intravenous transfection of antisense or sense ODN in mice on a high cholesterol diet, given the susceptibility of
C57/B6 mice to the development of atherosclerosis in response to a high cholesterol diet. A single systemic administration of antisense ODN increased serum cholesterol and triglyceride levels as compared with sense ODN, similar to mice on a normal diet (Fig. $4 \mathrm{a}-\mathrm{c}$ ).

Given the transient increase in cholesterol in antisense ODN-transfected mice, it is necessary to achieve longterm inhibition of apo $\mathrm{E}$ in order to study lipoprotein metabolism. Therefore, we hypothesize that repeated injection of antisense ODN would prolong the increase in cholesterol levels. Indeed, an increase in cholesterol levels was observed in antisense ODN-transfected mice for up to 24 days after the last transfection by cumulative injection (days 0, 1 and 2) (Fig. 5). Alternatively, we examined whether repeated injection of antisense ODN would achieve the same effect on the increase in cholesterol levels. As shown in Fig. 6a and b, repeated injection of antisense apo E ODN on day 7 and day 16 after the first injection resulted in a sustained increase in cholesterol and triglyceride levels for up to 23 days. Finally, we tried to maintain a high serum cholesterol concentration by systemic administration of antisense apo E ODN. Our present study showed the successful establishment of high cholesterol mice by local targeting of hepatic apo E with antisense ODN, as shown in Fig. 7a and b. After stopping injection of antisense apo E ODN, the high cholesterol levels gradually returned to the same levels as those in sense ODN-transfected mice.

\section{Discussion}

Atherosclerosis is a major cardiovascular risk factor for myocardial infarction and cerebrovascular disease. Although the pathophysiology of atherosclerosis is quite complicated, it is unquestionable that abnormality of lipid metabolism such as a high cholesterol level causes lipid 


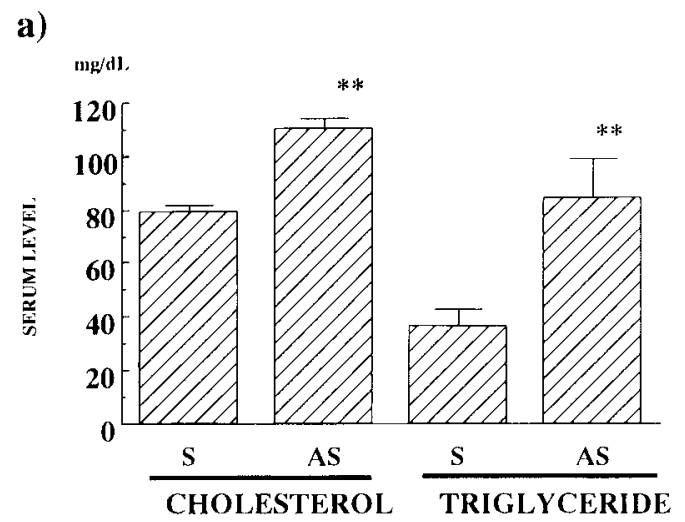

b)

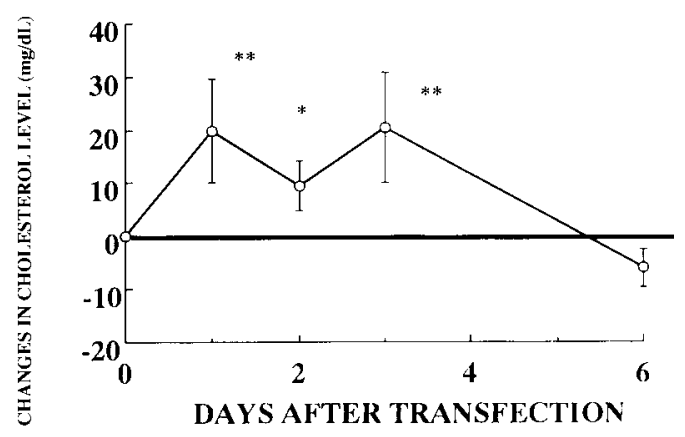

c)

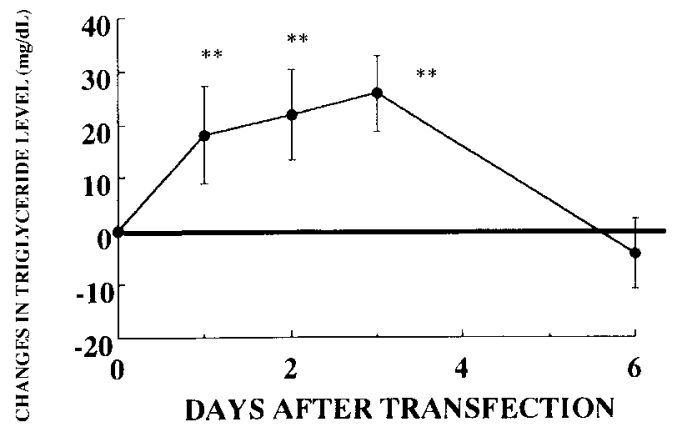

Figure 3 (a) Effect of antisense apo E ODN transfection on plasma cholesterol and triglyceride levels in mice on a normal diet at 3 days after transfection. $\mathrm{S}$, mice transfected with sense ODN; AS, mice transfected with antisense ODN. ${ }^{* *} P<0 \cdot 01 \mathrm{vs} \mathrm{S}$. (b and c) Time-course of changes in levels of (b) cholesterol and (c) triglyceride in antisense apo E ODN-transfected mice (normal diet). ${ }^{*} P<0 \cdot 05,{ }^{* *} P<0 \cdot 01$ vs mice transfected with sense ODN. Each group contained 6 animals. Values are antisense ODN - sense ODN.

deposition in the vascular wall. Especially, apolipoproteins such as apo E and apo (a) have an important role in the development of atherosclerosis (Lawn et al. 1992, Plump

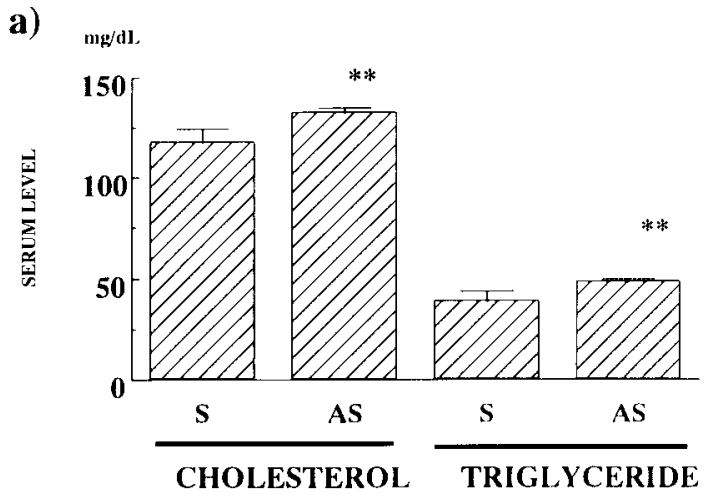

b)

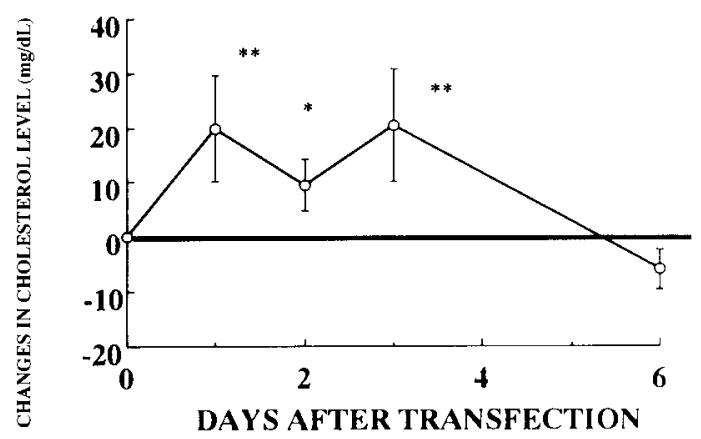

c)

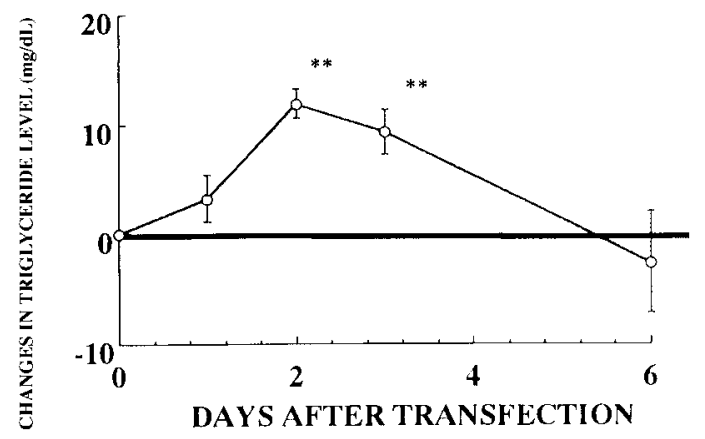

Figure 4 (a) Effect of antisense apo E ODN transfection on plasma cholesterol and triglyceride levels in mice on a high cholesterol diet at 3 days after transfection. $S$, mice transfected with sense ODN; AS, mice transfected with antisense ODN. ${ }^{* *} P<0 \cdot 01$ vs $S$. (b and c) Time-course of changes in levels of (b) cholesterol and (c) triglyceride in mice transfected with antisense apo E ODN (high cholesterol diet). ${ }^{*} P<0 \cdot 05,{ }^{*} P<0 \cdot 01$ vs mice transfected with sense ODN. Each group contained 6 animals. Values are antisense ODN - sense ODN.

et al. 1992, Zhang et al. 1992). It is well known that the plasma apo (a) level, which is determined by genotype, is an independent risk factor for myocardial infarction (Wade 


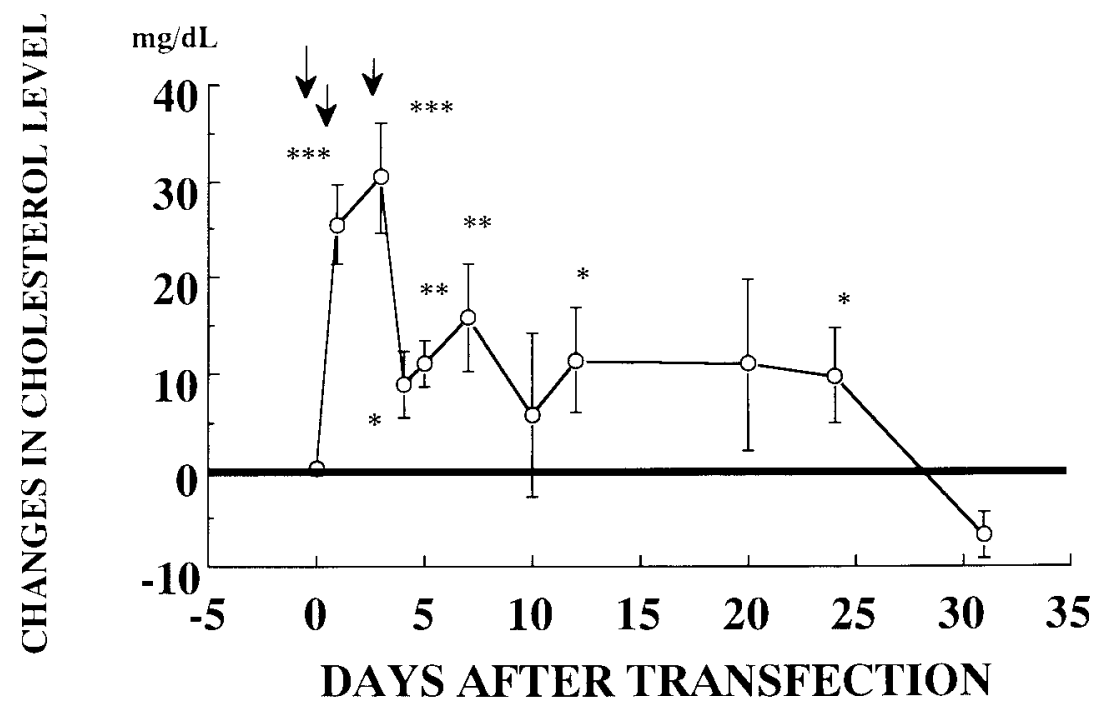

Figure 5 Effect of cumulative injection of antisense apo E ODN on cholesterol level (high cholesterol diet). ${ }^{*} P<0 \cdot 05,{ }^{* *} P<0 \cdot 01$ vs mice transfected with sense ODN. Each group contained 6 animals. Values are antisense ODN - sense ODN. Arrows show transfection.

et al. 1993). Apo $\mathrm{E}$ is also at the center of interest, because patients with apo E deficiency have a lipoprotein pattern resembling that seen in type III hyperlipoproteinemia and also develop atherosclerosis and xanthomas (Ghiselli et al. 1981, Schaeffer et al. 1986, Mabuchi et al. 1989, Mahley \& Rall 1989). Moreover, a common genetic variation of apo $\mathrm{E}$ in human populations is also known (Zannis et al. 1982).

Recent progress in molecular biology has enabled the study of the specific functions of lipoprotein genes. Using transgenic technology, overexpression or tissue-specific expression of lipoprotein genes can be analyzed. Alternatively, the complete inhibition of a gene can also be performed using gene targeting technology. Indeed, these technologies are quite powerful and useful for the study of lipoprotein metabolism. For example, overexpression of apo $\mathrm{E}$ prevented the development of atherosclerosis (Shimano et al. 1992). In contrast, mice lacking apo E showed spontaneous development of hypercholesterolemia and arterial lesions (Plump et al. 1992, Zhang et al. 1992). However, the transgenic/gene targeting technology has several disadvantages: (1) it is time consuming and costly, (2) the effect of the over-expressed transgene is exerted throughout development, and (3) it is difficult to exclude the potential contribution of the systemic effect of transgene expression. If the fate of knockout mice lacking the targeted gene is lethal, it is difficult to test the specific functions by transgenic or gene targeting techniques. In those cases, the gene transfer approach may be an alternative method.

Apo $\mathrm{E}$ is synthesized primarily in the liver, although synthesis has been documented in virtually every tissue. Therefore, hypercholesterolemia in apo E-deficient mice a)

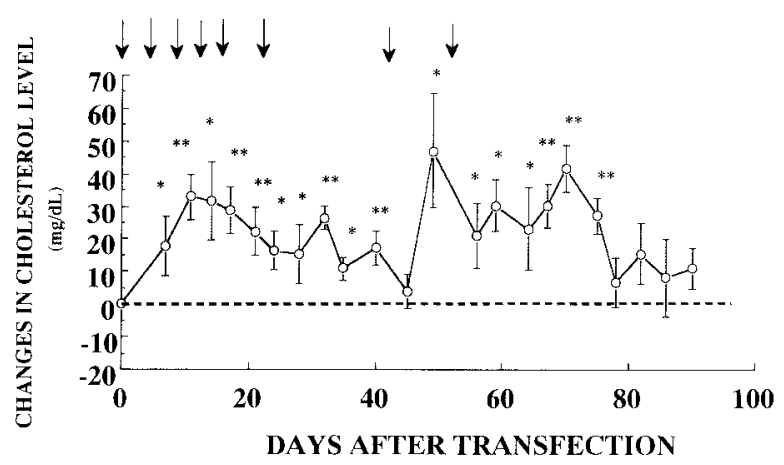

b)

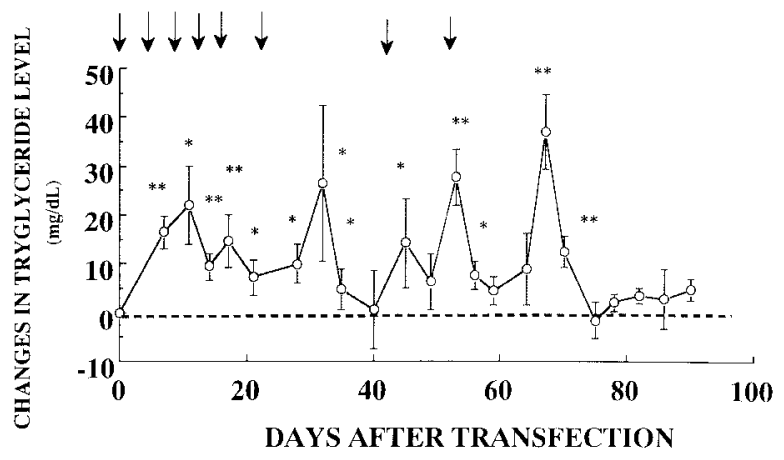

Figure 6 Effect of repeated injection of antisense apo E ODN on cholesterol (a) and triglyceride (b) levels (high cholesterol diet). ${ }^{*} P<0 \cdot 05,{ }^{* *} P<0 \cdot 01$ vs mice transfected with sense ODN. Each group contains 6 animals. Values are antisense ODN - sense ODN. Arrows show transfection. 
a)

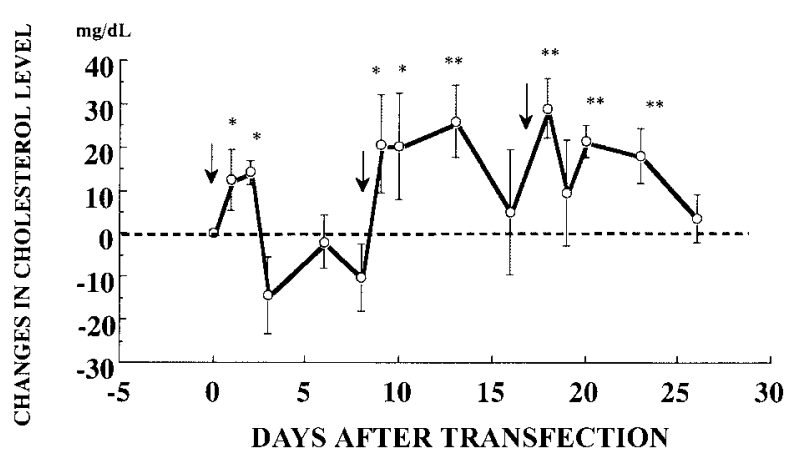

b)

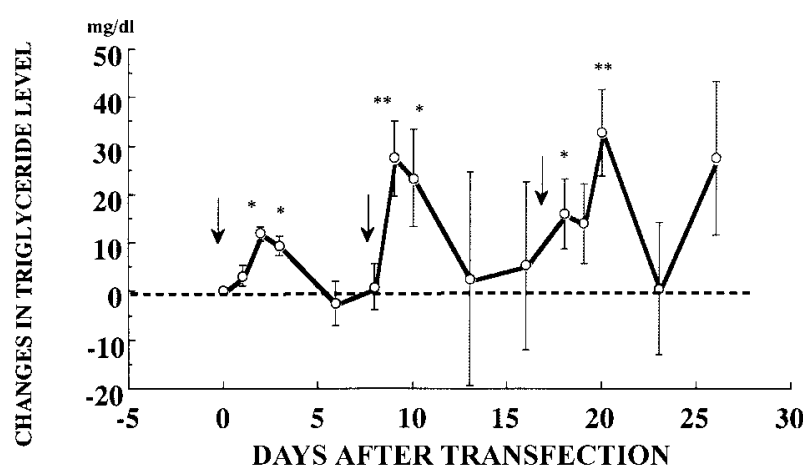

Figure 7 Effect of multiple repeated injection of antisense apo $\mathrm{E}$ ODN on cholesterol (a) and triglyceride (b) levels (high cholesterol diet). ${ }^{*} P<0 \cdot 05,{ }^{*} P<0 \cdot 01$ vs mice transfected with sense ODN. Each group contained 6 animals. Values are antisense ODN - sense ODN. Arrows show transfection.

created by gene targeting may not reflect the deficiency of hepatic apo E. The specific inhibition of hepatic apo E would be useful to study the role of apo E. To create hepatic apo E-deficient mice, we examined the systemic administration of antisense apo E ODN. Towards this aim, ODN were administered intravenously via the tail vein. However, passive uptake of ODN resulted in little accumulation in the liver and spleen, and also a short half life (less than 1 day). Next, we transfected ODN in liposomes (without HVJ), given the utility of liposomemediated delivery. Indeed, transfection of ODN in liposomes resulted in strong accumulation in the liver and spleen, but the short half-life could not be avoided (fluorescence disappeared within 1 day). Finally, we employed the HVJ-liposome method as the delivery system of ODN. As shown in this study, ODN in the HVJ-liposome complex could be transfected selectively into the liver, spleen and renal tubular cells. The localization of ODN in the HVJ-liposome complex was very different from that with passive uptake. Moreover, the
HVJ-liposome method resulted in marked prolongation of the half-life of ODN with persistence for at least 2 weeks after transfection. Given the unique character of the HVJ-liposome method, we transfected antisense apo E ODN in the HVJ-liposome complex by systemic injection.

Systemic administration of antisense apo E ODN resulted in selective inhibition of apo E mRNA expression in the liver, while there was no change in mRNA levels of apo $B$ and $\beta$-actin. In apo E-deficient mice created with antisense ODN, a transient increase in cholesterol and triglyceride levels could be observed as compared with sense ODN-treated mice. The same phenomenon was also observed in mice fed a high cholesterol diet. The specificity of antisense ODN was also supported by the observation that neither scrambled nor mismatched ODN resulted in an increase in cholesterol and triglyceride. However, the magnitude and duration of the increase in cholesterol did not seem sufficient to study the specific role of apolipoprotein, especially compared with transgenic or knock-out mice. To address this issue, the cumulative effects of transfection of antisense ODN were examined. The cumulative transfection of antisense apo E ODN resulted in a relatively long-term increase in cholesterol (up to day 24). Alternatively, repeated transfection of antisense apo E ODN was also performed. An increase in cholesterol and triglyceride was observed in response to every injection of apo E ODN.

Finally, we tried to maintain a sustained increase in cholesterol and triglyceride. Repeated systemic injections of antisense apo E ODN created apo E-deficient mice who showed a sustained increase in cholesterol and triglyceride over 3 months. The magnitude of the increase in cholesterol $(49 \mathrm{mg} / \mathrm{dl}$; from 137 to $186 \mathrm{mg} / \mathrm{dl}$ ) was relatively small as compared with that in homozygous gene targeted mice (cholesterol; from 132 to $1821 \mathrm{mg} / \mathrm{dl}$ ), but more severe than that in heterozygotes $(9 \mathrm{mg} / \mathrm{dl}$; from 132 to $141 \mathrm{mg} / \mathrm{dl}$ ). Reflecting the small increase in cholesterol level, the abdominal aorta and brachiocephalic artery showed no evidence of atherosclerotic lesions in both sense and antisense ODN-treated mice (data not shown). Interestingly, the magnitude of the increase in triglyceride level was similar to that in homozygous apo E-deficient mice (antisense $35 \mathrm{mg} / \mathrm{dl}$ versus homogygote $27 \mathrm{mg} / \mathrm{dl}$ ). Thus, hepatic apo E may play an important role in triglyceride metabolism. Nevertheless, the increase in plasma cholesterol was not dramatic, probably due to the low amounts of ODN or the short period of the inhibitory effects of antisense ODN. The limitation of the present study to create a new animal model is clearly apparent considering the transient change in the targeted genes rather than permanent inhibition. Further modification of ODN such as the change of ODN backbone to increase the resistance to nuclease would resolve these issues.

Alternatively, in vivo systemic administration of antisense ODN against lipoprotein may be considered as a 
gene therapy strategy for atherosclerosis. Lipoprotein (a) and apolipoprotein A-II are reported as independent risk factors for coronary artery disease (Davignon et al. 1988, Rubin et al. 1991, Abbey 1992, Wade et al. 1993, Morishita et al. 1994c). These lipoproteins are mainly synthesized in the liver, as for apo E. It is noteworthy that there is no pharmacotherapy to decrease such high risk lipoproteins selectively. We previously reported that ribozyme selectively induces cleavage of apo (a) without affecting plasminogen gene expression (Morishita et al. 1998). Together with the delivery system presented in this study, systemic administration of ribozyme against apo (a) may be useful to treat atherosclerosis induced by high lipoprotein (a). Systemic delivery of antisense or ribozyme by the HVJ-liposome method may provide the opportunity to treat such disease status. Moreover, this study addressed another potential problem, antigenicity of the viral vector system. We observed that the biological effects exerted by administration of antisense ODN were still reproduced following 8 repeated injections. Thus, it is unlikely that the HVJ-liposome method causes antigenicity. Indeed, we could not detect significant titers of antibody against HVJ after multiple repeated injections (Morishita et al. 2000). The low antigenicity of HVJliposome is consistent with a previous report (Hirano et al. 1998). Therefore, the simultaneous inhibition of double genes may be possible using the HVJ-liposome method, since we did not address this possibility in the present study. This character seems to be ideal for a future gene therapy strategy, although further characterization is needed. Overall, this study shows the feasibility of the in vivo antisense strategy to improve hyperlipoproteinemic metabolism.

In conclusion, systemic administration of antisense apo E ODN in the HVJ-liposome complex created hepatic apo E-deficient ('antigenic') mice which showed a transient increase in cholesterol and triglyceride. Repeated injection of antisense apo E ODN maintained apo E deficiency in mice over 3 months. Although apolipoprotein-deficient mice created by intravenous administration of antisense ODN seem to be a promising new animal model to help understand the role of apolipoprotein in vivo and develop new drug therapies targeting apolipoprotein, further development of ODN delivery methods and ODN backbone might be necessary.

\section{Acknowledgements}

This work was partially supported by a grant from a Grant-in-Aid from the Ministry of Public Health and Welfare, a Grant-in-Aid for the Development of Innovative Technology, a Grant-in-Aid from Japan Promotion of Science, and Special Coordination Funds of the Ministry of Education, Culture, Sports, Science and Technology, the Japanese Government.

\section{References}

Abbey M 1992 The influence of apolipoprotein polymorphism on the response to dietary fat and cholesterol. Current Opinion in Lipidology 3 12-16.

Davignon J, Gregg RE \& Sing CF 1988 Apolipoprotein E polymorphism and atherosclerosis. Arteriosclerosis 8 1-21.

Dzau VJ, Gibbons GH, Morishita R \& Pratt RE 1994 New perspectives in hypertension research: potentials of vascular biology. Hypertension 23 1132-1140.

Ghiselli G, Schaeffer EJ, Gascon P \& Brewer HB Jr 1981 Type III hyperlipoproteinemia associated with apolipoprotein E deficiency. Science 214 1239-1241.

Hirano T, Fujimoto J, Ueki T, Yamamoto H, Iwasaki T, Morishita R, Sawa Y, Kaneda Y, Takahashi H \& Okamoto E 1998 Persistent gene expression in rat liver in vivo by repetitive transfections using HVJ-liposome. Gene Therapy 5 459-464.

Kittelberger R, Davis PF \& Stehbens WE 1989 An improved immunofluorescence technique for the histological examination of blood vessel tissue. Acta Histochemica 86 137-142.

Lawn RM, Wade DP, Hammer RE, Chiesa G, Verstuyft JG \& Rubin EM 1992 Atherogenesis in transgenic mice expressing human apolipoprotein (a). Nature 360 670-672.

Mabuchi H, Itoh H, Takeda M, Kajinami K, Wakasugi T, Koizumi J, Takeda R \& Asagami C 1989 A young type III hyperlipoproteinemic patient associated with apolipoprotein E deficiency. Metabolism 38 115-119.

Mahley RW 1988 Apolipoprotein E: cholesterol transport protein with expanding role in cell biology. Science 240 622-630.

Mahley RW \& Rall SC Jr 1989 Type III hyperlipoproteinemia (dysbetalipoproteinemia): the role of apolipoprotein $\mathrm{E}$ in normal and abnormal lipoprotein metabolism. In The Metabolic Basis of Inherited Disease, edn 6, pp 1195-1213. Eds CR Scriver, AL Beaudet, WS Sly \& D Valle. New York: MacGraw Hill.

Morishita R, Higaki J, Miyazaki M \& Ogihara T 1992 Possible role of the vascular renin angiotensin system in hypertension and vascular hypertrophy. Hypertension 19 II-62-67.

Morishita R, Gibbons GH, Ellison KE, Nakajima M, Zhang L, Kaneda Y, Ogihara T \& Dzau VJ 1993 Single intraluminal delivery of antisense cdc 2 kinase and PCNA oligonucleotides results in chronic inhibition of neointimal hyperplasia. PNAS $\mathbf{9 0}$ 8474-8478.

Morishita R, Gibbons GH, Ellison KE, Lee W, Zhang L, Kaneda Y, Ogihara T \& Dzau VJ 1994a Evidence for direct local effect of angiotensin in vascular hypertrophy: in vivo gene transfer of angiotensin converting enzyme. Journal of Clinical Investigation 94 978-984.

Morishita R, Gibbons GH, Ellison KE, Nakajima M, Leyen HVL, Zhang L, Kaneda Y, Ogihara T \& Dzau VJ $1994 b$ Intimal hyperplasia after vascular injury is inhibited by antisense $\mathrm{cdk} 2$ kinase oligonucleotides. Journal of Clinical Investigation 93 $1458-1464$

Morishita R, Gibbons GH, Kaneda Y, Ogihara T \& Dzau VJ 1994c Pharmacokinetics of antisense oligonucleotides (cyclin B1 and cdc 2 kinase) in the vessel wall: enhanced therapeutic utility for restenosis by HVJ-liposome method. Gene 149 13-19.

Morishita R, Gibbons GH, Horiuchi M, Nakajima M, Ellison KE, Lee W, Kaneda Y, Ogihara T \& Dzau VJ 1996 Molecular delivery system for antisense oligonucleotides: enhanced effectiveness of antisense oligonucleotides by HVJ-liposome mediated transfer. Journal of Cardiovascular Pharmacology and Therapentics 2 213-222.

Morishita R, Yamada S, Yamamoto K, Tomita N, Kida I, Sakurabayashi I, Kikuchi A, Kaneda Y, Lawn R, Higaki J \& Ogihara T 1998 Novel therapeutic strategy for atherosclerosis: ribozyme oligonucleotides against apolipoprotein (a) selectively inhibit apolipoprotein (a), but not plasminogen, gene expression. Circulation 98 1898-1904. 
Morishita R, Gibbons GH, Kaneda Y, Ogihara T \& Dzau VJ 2000 Systemic administration of HVJ viral coat-liposome complex containing human insulin vector decreases glucose level in diabetic mouse: a model of gene therapy. Biochemical and Biophysical Research Communications 273 666-674.

Plump AS, Smith JD, Hayek T, Aalto-Setala K, Walsh A, Verstuyft JG, Rubin EM \& Breslow JL 1992 Severe hypercholesterolemia and atherosclerosis in apolipoprotein E-deficient mice created by homologous recombination in ES cells. Cell 71 343-353.

Rubin RE, Krauss RM, Spangler EA, Verstuyft JG \& Clift SM 1991 Inhibition of early atherogenesis in transgenic mice by human apolipoprotein A-1. Nature 353 265-267.

Schaeffer EJ, Gregg RE, Ghiselli G, Forte TM, Ordovas JM, Zech LA \& Brewer HB Jr 1986 Familial apolipoprotein E deficiency. Journal of Clinical Investigation 78 1206-1219.

Shimano H, Yamada N, Katsuki M, Shimada M, Gotoda T, Harada K, Murase T, Fukazawa C, Takaku F \& Yazaki Y 1992 Overexpression of apolipoprotein $\mathrm{E}$ in transgenic mice: marked reduction in plasma lipoproteins except high density lipoprotein and resistance against diet-induced hypercholesterolemia. PNAS $\mathbf{8 9}$ $1750-1754$

Tomita N, Higaki J, Kaneda Y, Yu H, Morishita R, Mikami H \& Ogihara T 1993 Hypertensive rats produced by in vivo introduction of the human renin gene. Circulation Research $\mathbf{7 3}$ 898-905.

Utermann G, Steinmetz A \& Weber W 1982 Genetic control of human apolipoprotein E polymorphism: comparison of one- and two-dimensional techniques of isoprotein analysis. Human Genetics $60344-351$.
Wade DP, Clarke JG, Lindahl GE, Liu AC, Zysow BR, Meer K, Schwartz K \& Lawn RM 1993 Genetic influences on lipoprotein (a) concentration. Biochemical Society Transactions 21 499-502.

Wang AM, Doyle MV \& Mark DF 1989 Quantitation of mRNA by the polymerase chain reaction. PNAS 86 9717-9721.

Weintrab MS, Eisenberg S \& Breslow JL 1987 Different patterns of postprandial lipoprotein metabolism in normal, type IIa, type III, and type IV hyperlipoproteinemic individuals. Journal of Clinical Investigation 79 1110-1119.

Xiong W, Zsigmond E, Gotto AM Jr, Reneker LW \& Chan L 1992 Transgenic mice expressing full-length human apolipoprotein B-100. Full-length human apolipoprotein B mRNA is essentially not edited in mouse intestine or liver. Journal of Biological Chemistry 267 21412-21420.

Zannis VI \& Breslow JL 1981 Human very low density lipoprotein apolipoprotein $\mathrm{E}$ isoprotein polymorphism is explained by genetic variation and posttranslational modification. Biochemistry $\mathbf{2 0}$ 1033-1041.

Zannis VI, Breslow JL, Utermann G, Mahley RW, Weisgraber KH, Havel RJ, Goldstein JL, Brown MS, Schonfeld G, Hazzard WR \& Blum CB 1982 Proposed nomenclature of apo E isoprotein genotypes and phenotypes. Journal of Lipid Research 23 911-914.

Zhang SH, Roddick RL, Piedrahita JA \& Maeda N 1992 Spontaneous hypercholesterolemia and arterial lesions in mice lacking apolipoprotein E. Science 258 468-471.

Received 24 June 2002

Accepted 23 July 2002 